G587(P) MEASLES ANTIBODY LEVELS AMONG VACCINATED HIVINFECTED AND HIV UNINFECTED CHILDREN IN NIGERIA

${ }^{1}$ BO Olorunsola, ${ }^{2}$ OT Adedoyin, ${ }^{2}$ SK Ernest. 'Children Services, Scunthorpe General Hospital, Northern Lincolnshire and Goole NHS FT, Scunthorpe, UK; ${ }^{2}$ Paediatrics and Childhealth, University of Ilorin Teaching Hospital, Ilorin, Nigeria

\subsection{6/archdischild-2020-rcpch.504}

Background Sub-Saharan African countries overwhelmingly bear the burden of global HIV infection and measles outbreaks in children. Despite the significant impact of this double burden in Nigeria, the effect of HIV infection on measles antibody levels among children is unknown. This study was therefore conducted to compare the measles antibody levels among HIV infected and uninfected children.

Methods The study was a descriptive comparative cross-sectional study among $180 \mathrm{HIV}$ infected and uninfected children aged 2-10 year, recruited between August and December 2015 in a Tertiary Healthcare centre in Nigeria. Socio-demographic, clinical and anthropometric parameters were obtained. Blood samples were collected for haematologic evaluation (CD4+ cell count, full blood count) and serologic assay of measles antibody using IMMUNOLAB ELISA kit. Data was analyzed using SPSS version 20.

Results A total of $90 \mathrm{HIV}$ infected subjects and 90 age and sex-matched HIV-negative controls were analyzed. There were 48 males and 42 females aged between 2 to 10 years with a Male: Female ratio of $1.1: 1$ in both groups. The mean age was 5.4 years.

While the seroprevalence of measles antibody was 46.7\% among the HIV negative children, only 9\% of the HIV infected subjects had positive antibody level. The antibody titre was also significantly lower among HIV infected subjects compared with controls with median measles antibody values of $3.3 \mathrm{U} / \mathrm{ml}$ and $9.4 \mathrm{U} / \mathrm{ml}$ respectively ( $p$ $<0.001$ ).

HIV infected subjects with more than one dose of measles vaccine had significantly higher seroprevalence of measles antibody than those with single dose $(38.5 \%$ vs $5.2 \%$, p $<0.003)$.

Conclusion There was a low seroprevalence of measles antibody among vaccinated HIV infected children in Nigeria, with higher seroprevalence of measles antibody among children with more than one (1) dose of measles vaccine. It is therefore advocated that HIV infected children should be given additional dose(s) of measles vaccination.

\section{G588(P) SCRUB TYPHUS: A SERIES OF CASES IN A TERTIARY CARE CENTRE FROM APRIL 2018 TO JULY 2019}

${ }^{1} \mathrm{G}$ Sahariah Khound, ${ }^{1} \mathrm{R}$ Khaund Borkotoky, ${ }^{1} \mathrm{AL}$ Saha, ${ }^{1} \mathrm{P}$ Banerjee Barua, ${ }^{1} \mathrm{~B}$ Das, ${ }^{2} \mathrm{~N}$ Khound, 'S Pathak. 'Paediatrics, Apollo Hospital Guwahati, Guwahati, India; ${ }^{2}$ Medicine, Apollo Hospital Guwahati, Guwahati, India

\subsection{6/archdischild-2020-rcpch.505}

Aim Although Scrub Typhus is an endemic disease, it is grossly underdiagnosed owing to the nonspecific clinical presentation, lack of access to specific diagnostic facilities in most areas and low index of suspicion by the clinician. Our aim of the study is to increase the awareness of the disease amongst the clinicians.

Methods Seven patients with acute febrile illness diagnosed to have scrub typhus are reported here with varied presentations from April 2018 to July 2019. First case presented with fever, headache, cough with shortness of breath in the month of April 2018. Second case presented with fever with chills and rigors, cough, respiratory difficulty, passage of dark coloured urine in May 2018. Third case presented with fever, breathing difficulty, multiple eschars, two episodes of cardiac arrest and vomiting in Sept 2018. Fourth case presented with fever, pain abdomen, breathing difficulty, passage of black coloured stool in May 2019. Fifth case presented with fever with chills and rigors, cough, erythematous rashes over face, hands, legs and body and an eschar on left axillae in May 2019. Sixth case a six month old infant with fever and rashes all over the body, eschar on left leg and passage of loose stool in the month of June 2019. Seventh case presented with fever with breathing difficulty, yellowish discoloration of urine, generalized oedema, in the month of July 2019. To diagnose the cases we used immunochromatographic ( $\operatorname{IgM}$ and $\operatorname{IgG}$ ) study for scrub typhus. Due to resource constraints we couldn't use Immunofluorescence Antibody ( IFA ) to confirm the diagnosis. Doxycycline with other supportive medications rapidly altered the clinical course without any sequelae.

Results All the patients responded well to Doxycycline and other supportives. Defervescence occurred in 24 to 48 hours of using first dose of Doxycycline.

Conclusion High index of suspicion, timely diagnosis and appropriate treatment can reduce the morbidity and mortality of Scrub Typhus.

\section{G589(P) CHILDHOOD TUBERCULOSIS STILL A SIGNIFICANT BURDEN: EXPERIENCE OF A DISTRICT DEDICATED TUBERCULOSIS SERVICE}

TP Newson, O Smith. Paediatric Department, East Kent Hospital University NHS FT, Canterbury, UK

\subsection{6/archdischild-2020-rcpch.506}

Aims Evaluate our experience of a dedicated Tuberculosis service for children in a district general setting.

Methods Rates of Tuberculosis (TB) in our area in the South East are the second highest in England and our Trust covers areas of significant deprivation. There became a need to set up a dedicated service for childhood TB in partnership with our TB community nursing team and close liaison with adult respiratory colleagues. We started in 2005 to run a monthly clinic with 2 consultants with training in $\mathrm{TB}$ and a dedicated TB Nurse a unique service in the South. We analysed retrospectively our recent experience 2017 to 2018.

Results 10 cases of active TB were identified,age range 11 weeks to 16 years, 6 Caucasian, 2 Nepalese and 2 Eritrean. There were 37 cases latentTB age range 1 month to 16 years, 14 Caucasian, 15 Nepalese and 8 Indian. The active cases all had pulmonary TB with 100\% cough, 50\% haemoptysis,30\% fever,20\% night sweats, $20 \%$ weight loss. History of contact with smear positive adult in $70 \%$ and foreign travel $40 \%$. Chest X Ray changes 70\%: 2 RUL, 3 RLL, Pleural effusion, 1 hilar nodes. ESR raised 40\%,Mantoux positive 90\%,T Spot positive $70 \%$, AAFBs or culture TB 20\%. All HIV negative. All successfully treated using DOT and VOTs. Use of Latent TB government video for explaining latent $\mathrm{TB}$ was popular. All cases were discussed with Adult TB team and clinic visits always had TB nurse present. 
Conclusions In areas with high rates of $\mathrm{TB}$ in a district a dedicated district service seems an effective way to see patients within a Mutidiscliplinary Team and allows good liason with Adult TB team. The setting up of networks for Paediatric TB may encourage this set up with discussion of active cases with tertiary infectious disease specialists while maintaining good local supportive supervision and deliver of care.

\section{G590(P) THE PREVALENCE OF HYPOCALCEMIA IN CHILDREN WITH DENGUE INFECTION: A 1 YEAR CROSS-SECTIONAL STUDY}

Miss Singh, Mr Kamble. Paediatrics, JNMC, Belagavi, India

10.1136/archdischild-2020-rcpch.507

Introduction According to WHO, Dengue is fast emerging pandemic-prone viral disease affecting mainly urban poor areas in tropical and subtropical countries. There is an urgent need for early recognition and prompt treatment in order to limit the morbidity and mortality. One of the important electrolyte disturbance documented in critical phase of severe dengue is hypocalcaemia. Studies have linked calcium with the immune response and severity of infection of dengue virus.

Objectives

- To study the prevalence of hypocalcaemia in children suffering from dengue infection.

- To study the association between the degree of hypocalcaemia and the severity of the illness.

Methods and Material This study is a one-year cross sectional observational study, conducted in a tertiary care hospital. 100 subjects aged 1 month to 18 years suffering from dengue were included in the study. The subjects were classified as per 2009 WHO classification into Dengue fever without warning sign, Dengue fever with warning signs (DWS) and Severe dengue (SD). Ionic calcium and other biochemical parameters were done for all the subjects within 24 hours of admission. The clinical and biochemical data was coded and analyzed.

Results The mean age of the study population was $9.65 \mathrm{yr}$ with $58 \%$ males and $42 \%$ females. The prevalence of hypocalcemia was found to be $59.0 \%$. The mean $\mathrm{iCa}$ was 1.05 $\mathrm{mmol} / \mathrm{L}$. The study showed that $11.7 \%$ subjects with dengue without warning signs, $83.7 \%$ subjects of DWS and $100 \%$ of the subjects of SD had hypocalcaemia. It was seen that level of $\mathrm{iCa}$ is lowest in severe dengue $(\mathrm{p}<0.001)$.

$\begin{aligned} & \text { Abstract } \\
& \text { iCa }\end{aligned}$
\begin{tabular}{lllllll}
\hline Variables & Table 1 & Distribution of subjects with respect to \\
\hline iCa (mmol/L) & Classification of dengue & N & Mean iCa & Std. Dev & F & Sig. \\
& Dengue with warning signs & 43 & 1.05 & 0.06 & & \\
& Severe dengue & 19 & 0.98 & 0.05 & & \\
\hline
\end{tabular}

Conclusion This study proves the association of the hypocalcaemia with the severity of dengue infection in the paediatric population and potentiates the use of ionic calcium as a biochemical marker to detect severe dengue infection early.

\section{G591(P) ARE WE LOSING THE BATTLE AGAINST INFECTIONS? AN AUDIT ON ANTIMICROBIAL RESISTANCE}

${ }^{1} \mathrm{~L}$ Rehman, ${ }^{2} \mathrm{M}$ Kamal, ${ }^{1} \mathrm{~F}$ Neenan, ${ }^{3} \mathrm{M}$ Hussain, ${ }^{1} \mathrm{M}$ Shahid. ${ }^{1}$ Paediatrics Department, Portiuncula University Hospital, Ballinasloe, Ireland; ${ }^{2}$ Paediatric Emergency Department, Tallaght University Hospital, Dublin, Ireland; ${ }^{3}$ Department of Children and Young People, North Cumbria Integrated Care, Whitehaven, UK

\subsection{6/archdischild-2020-rcpch.508}

Aim To analyse the practice of prescribing outpatient antibiotics and check adherence to antimicrobial guidelines in the Paediatric Emergency Department (PED) of a university hospital. Method All children, prescribed antibiotics before discharge from PED were included. Pre-intervention, 55 charts in between Feb-April 2019 were audited retrospectively for prescribing 1)Correctly indicated antibiotics against certain infections based on clinical judgement and/or investigations, 2) Correct choice, 3)Duration and 4)Dose against standards as

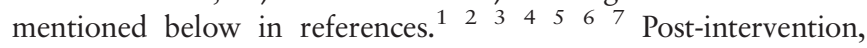
30 charts were audited between May-July 2019 to re-check adherence to recommended standards. Standards were redefined and implemented for common infections encountered in PED by physicians as per results of the audit. Clinical judgment was deemed optimal if it followed the standard. ${ }^{1} 2334567$

Results Pre interventions, only $56 \%$ of antibiotics were correctly indicated for certain infections. Post intervention, 96\% were correctly indicated, resulting in an improvement of $20 \%$. Regarding prescribing the correct type of antimicrobials, antibiotic guidelines were adhered to $84 \%$ of the time. This rose to $100 \%$ post-intervention. Of the antibiotics prescribed, $41 \%$ of outpatients were prescribed an insufficient dose which declined to $3 \%$ after intervention.

Conclusion Despite, substantially reduced mortality from infectious diseases by advances in antimicrobials, resistance is growing at an alarming rate. This rise in antimicrobial resistance is one of the greatest potential threats to human health at global levels with serious consequences for public health, animal health and welfare. ${ }^{8} 9$ Outpatient antimicrobial scripts are being written in the PED on a daily basis by junior doctors who rotate from different specialities every six months. Also, factors such as sub-optimal clinical judgement, parental reassurance and heavy workload in PED might contribute to excessive prescription. Our audit prompted us to make standards clear for all NCHDS. Our interventions reduced the margin of error substantially.

\section{REFERENCES}

1. Childhood Pneumonia, Faculty of Paediatrics (RCPI), HSE Guidelines, Feb 2014

2. Urinary Tract Infection, Faculty of Paediatrics (RCPI), HSE Guidelines

3. Acute Otitis Media, Faculty of Paediatrics (RCPI), HSE Guidelines

4. Sore throat (acute): antimicrobial prescribing, National Institute for Health and Care Excellence(NICE) guidelines, 2018

5. Our Lady's Children Hospital Formulary, OLCHC/TSCUH ANTIBIOTIC GUIDELINES

6. Galway Antimicrobial Prescribing Principles(GAPP)

7. BNF For Children 2019

8. https://health.gov.ie/blog/publications/irelands-national-action-plan-on-antimicrobial-resistance-2017-2020/

9. https://www.who.int/antimicrobial-resistance/global-action-plan/en/

\section{G592(P) DISSEMINATED NEONATAL HERPES: THE DEADLY MASQUERADER}

V Subbaraju, S Pradhan, P Nair. Paediatric Department, Bedford Hospital NHS Trust, Bedford, UK

10.1136/archdischild-2020-rcpch.509 